\title{
Distributed Collaborative Design over Cave2 Framework
}

\author{
Leandro Soares Indrusiak ${ }^{1,2}$, Juergen Becker ${ }^{2}$, Manfred Glesner ${ }^{2}$, Ricardo \\ Augusto da Luz Reis ${ }^{1}$
}

1 Instituto de Informática, Universidade Federal do Rio Grande do Sul, Porto Alegre, Brazil
2 Institute of Microelectronic Systems, Technische Universität Darmstadt, Darmstadt, Germany

E-mail: <lsi,becker,glesner>@mes.tu-darmstadt.de, reis@inf.ufrgs.br

\begin{abstract}
This paper presents the research and development of mechanisms to allow distributed collaborative design of integrated systems. Those mechanisms were implemented over the software infrastructure developed under the Cave Project, taking advantage on both definitions of the framework concept: classical electronic design frameworks and object-oriented extensible data modeling. The final result is a design environment accessible over Internet-like networks, where groups of designers can work over the design representation in a collaborative way. In order to organize the interaction between the designers, an extension to the Pair Programming collaboration methodology was developed and implemented in a case study.
\end{abstract}




\section{INTRODUCTION}

Recent advances on the application of system level modeling techniques show that this approach is already mature to be considered for production environments $[1,2,3,4]$. However, the advantages granted by such approach depends strongly on how those concepts can be understood and adopted by the designers. Such kind of paradigm shift was already imposed to the designers, as a result of the pursuit of productivity boost by raising the abstraction levels of the design specification: physical layout, logic schematic diagrams, HDLs. In most of the cases, they are able to cope with such transitions by relying on new design environments, training sessions and support from the colleagues. And it seems that they are expected to handle the transition we're going through in the same way as they did before. Similar experiences were reported in software engineering, when the transition between the structured and the object-oriented paradigm took place.

Taking into account such transition scenario, new design automation infrastructure is needed to reduce the complexity of the new paradigm, making the transition as smooth as possible for the designers.

The work described in this paper is a part of the ongoing research of a design environment tailored for the needs of a designer without familiarity with system level concepts. The following points are key issues to make such design environment possible:

correlation between system level design concepts and the concepts which are familiar to the designer - e.g. structural or behavioral modeling using HDLs - through the interoperability of their graphical representations;

on-the-tool learning, where the designer can learn the new concepts by using the tool, guided by a remote tutor or pre-recorded usage scenarios;

remote collaboration, allowing beginners to learn from the experienced ones while working in a single project, even if the participants are in different locations.

In order to implement such ambitious proposal, the system is being built over the infrastructure of the Cave Project - a research effort started at UFRGS University in 1996, and which now includes other research groups in South America and Europe.

This paper has its main focus on the third point presented above - remote collaboration - but it will mention briefly some issues related to the other two concepts. It is organized as follows: in the next session, the Cave Project background is briefly presented, and its infrastructure is detailed; Section 3 reviews the constructs used for design modeling at system level; Section 4 details the chosen collaboration models, as well as the software architecture developed to integrate such model in the Cave2 Framework; Section 5 presents a case study for a collaborative design flow from a 
system level specification; finally, conclusions, open issues, future development and references are presented.

\section{CAVE PROJECT BACKGROUND}

As defined in [5], a CAD Framework is a software infrastructure aimed to support the development, interoperation and usage of design automation tools. The first release of the Cave Project, known as the Cave Framework [6,7], followed that guideline, emphasizing the possibility of the distribution of design automation resources over Internet using WWW and the HTTP protocol. A library of object-oriented primitives for GUI development was created, in order to enhance the functionality of web browsers, which were the main interface between the designer and the framework. A set of modules was also developed, easing the integration of design tools with HTTP servers.

While successful in some of its usage scenarios [8,9], two critical points inherited from the WWW architecture - lack of support for user awareness and file-based data transfer and storage - limited the possibilities of true distributed collaborative design. So, a new architecture was presented in [10] under the name Cave2 Framework, with a different approach to overcome the deficiencies (Figure 1). While still using the Internet as communication infrastructure, it focused more in the library of reusable object-oriented code and on the extensibility of that library through the concept of framework, but this time using the meaning from the software engineering domain. According to [11], a framework is a reusable software design expressed as a set of abstract classes and the way their instances collaborate. Based on such approach, the Cave2 Framework can provide an extensible set of design tool GUI primitives, which are used to assemble dynamically the interface between the user and the design environment according to the tasks he/she wants to perform. Furthermore, the software framework approach also allowed the modeling of design data using objects, in opposition to the file-based model used in the first version. So, relationships among those objects can be defined using patterns [12], keeping the semantic of those relationships on every instance of the object. This is a key issue to allow the correlation between system-level and structural concepts and - as detailed in the following sections - the implementation of richer collaboration models within the design environment.

Besides the organization of the class libraries in a framework, the Cave2 Framework also organized the server-side facilities. Formerly attachments to the HTTP server, now they are implemented also as objects and are 
executed in a Service Space, following the architecture proposed in [13]. Current services implemented in such space include persistence, authentication, integration with external tools and, as described in the next session, collaboration.

The Cave2 Framework can be executed over Internet/Intranet, using regular HTTP servers and regular Java-enabled web browsers. Design resources distribution can be easily done, because the Cave 2 architecture support the cooperation of several Framework Servers and Service Spaces. In both cases, a non-redundant approach was used. Every design representation or design tool module is stored in only one Framework Server, and a Intra-Server protocol is used to share such resources. Similarly, every service resides in only one Service Space, and the lookup mechanism described in [13] is used for searching for services among all the available spaces.

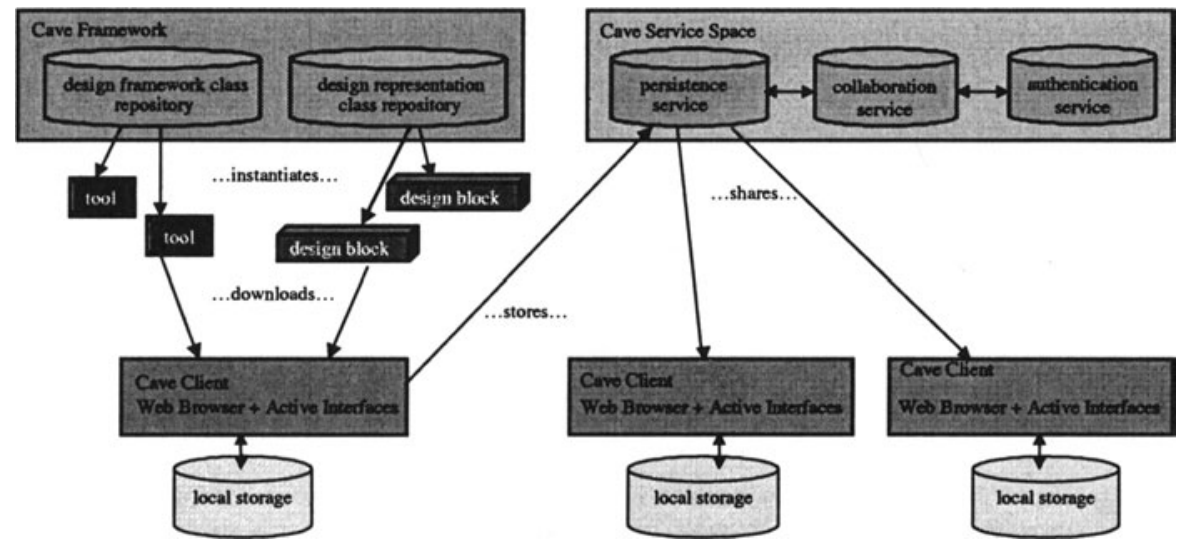

Figure 1. Cave2 Framework Architecture

\section{DESIGN MODELING CONSTRUCTS}

The recent advances on the research of system level modeling techniques is forcing a paradigm shift by the designers. New languages and modeling tools are being introduced, based on such techniques, requiring from the designers a different understanding of the design process. 
This scenario was already seen before, as new hardware design paradigms were introduced. If we review the evolution of the integrated circuits design procedure in the last thirty years, we notice that on every step taken to improve productivity - by making the design abstraction levels higher - a new kind of design perception was required by the designers: layout edition, logic schematic capture, FSMs, HDLs, among others.

The transition between paradigms by the designers is always difficult, and it has been supported by the evolution of the design environments.

The use of graphical representations of the design objects in such environments is one of the strategies to reduce the complexity of the design task - for circuit layout, logic schematic or FSM edition it seems obvious, but even HDL-based design environments often take advantage on some diagrammatic representation.

However, the current approaches for system level design environments don't have a common focus. The approaches based on extensions of programming languages - such as SpecC[1], SystemC[2], Forge[3], Ocapi[4] - motivate the use of IDE-like tools for source code maintenance without any graphical support other than file management GUI. In other hand, well defined visual modeling tools are also being used, such as UML[14] and SDL[15], requiring complex graphical support from the design environment.

In the Cave2 Framework, graphic and textual edition primitives are available for the designer to assemble his/her design entry tools, allowing both kinds of modeling for system level design, as well as lower abstraction design modeling through HDLs and logic schematic entry.

\section{DISTRIBUTED COLLABORATIVE DESIGN}

The need for distributed collaborative design can be easily justified:

- increasing complexity of designs, demanding larger teams;

- shortage of engineers, requiring manpower from different parts of the world;

- need for experience sharing among the members of a team, allowing the beginner to learn from the more experienced.

Some approaches for collaborative work support were presented previously in the EDA domain [16, 17], focusing IP core sharing, remote training or task-flow modeling. For the approach presented here, however, a different focus had to be found, because the collaborative model should be generic enough to support collaboration over different kinds of objects: texts and several kinds of diagrams.

The chosen solution used and extended the infrastructure provided by the Cave2 architecture: the support for collaborative work was included in the 
abstract classes within the design data representation framework, and the behavior of the collaborative sessions is controlled by a collaboration service in the Cave2 Service Space. The following subsections detail both parts of the solution.

\subsection{Collaboration-enabled design primitives}

In the Cave2 Framework, the design representation primitives - e.g. logic gates, functional blocks, etc. - are modeled as instances of a concrete class, which inherits behavior from an abstract class. This is common when it comes to object-oriented frameworks, because the abstract classes - while not modeling anything in the application domain - organize the class hierarchy and allow the assignment of common behavior to a particular set of objects. This concept was used to model all the support for the collaborative services: that support was included in the superclass of all the design primitive classes. So, all the design objects in the Cave2 Framework including the ones which will be integrated in future updates - will inherit such behavior.

Currently, the collaboration platform includes the following features:

- separation of concepts: the design semantic and its graphical/textual representation are modeled by different objects, in order to allow several visualizations - by different designers - from a single design block;

- update/notify mechanism: a 2-way update/notify mechanism was implemented, to grant consistency between the design semantic and its representations.

Using such features, several designers can work concurrently in a design. When two or more designers are working over a single design block, the object representing the block semantic is stored in the Cave Persistence Server, while a view of that block is instantiated for each one of the designers and stored on their GUIs. The interaction between the designer and the design block occurs through that view: when the view is modified in such way the design semantic changes, the view object notifies the block object in the Persistence Service, so it can update its state no reflect the change. Once the state is updated, a notification is done to every other view of that block, making the changes visible to the other users. It is important to note that when the interaction between the designer and the view is not changing the design semantic - e.g. when the designer moves a block in the design sheet without changing its interconnection with the other blocks - the update/notify mechanism is not activated. This approach is called visually decoupled (Figure 2a), because the view for each user can - and probably will - be different from the others. 
While allowing rich collaboration through the concurrent access to design blocks, this approach has the disadvantage of making impossible the spatial referencing of design blocks by the designers: one designer would fail to reference a particular block while communicating with another designer if he/she says e.g. "that FIFO in the left side of the encoder", because probably the blocks positioning in the screen of his/her colleague would be different. So, when spatial referencing is desired, we provide a visually coupled approach (Figure $2 b$ ), where the update/notify mechanism is slightly different. In this case there is only one view, stored together with the block in the Persistence Server. Every designer would have in his/her GUI a reference to that view, so every update - even the ones which are transparent to the design semantic - would be noticed by every designer.

\subsection{Collaboration service}

Taking into account the 1-to-n cardinality in the relationship of the design blocks with their views, it is easy to realize the need for an data access control mechanism. The possibilities of deadlock in the update/notify mechanism (i.e. when two views try to modify the semantic at the same time) and inconsistencies between the block and its views (i.e. when a view tries an update to the semantic before the reception of the notification of an update done previously) are some of the problems that may occur. The collaboration service performs such control, while providing a common interface for every tool in the Cave 2 environment, allowing them to use the design primitives in a collaborative way. Stored in the Cave Service Space and working together with the Authentication and Persistence Services, it uses a transaction-based approach to organize the access to the design objects by the users and to grant ACID properties: atomicity, consistency, isolation and durability.

In the top of such transaction-based mechanism, several collaboration models can be implemented.

\section{CASE STUDY}

\subsection{Collaboration Methodology}

The extensions to the Cave2 Framework described in this paper were implemented, as a case study, and a well known collaboration model was used in the first prototype of the Collaboration Service. The requirements 
for such collaboration model were the easy adaptation for the proposed transaction scheme and the suitability for collaboration over heterogeneous data representation. The chosen method for collaboration was the pair programming [18], a methodology mainly used for collaboration in software engineering. According to that method, the quality of the code developed by a programmer increases significantly when it is concurrently reviewed by a

(a)

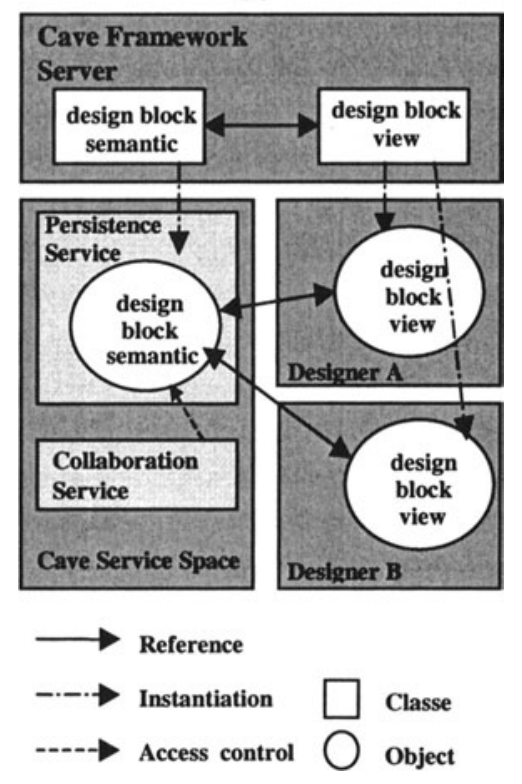

(b)

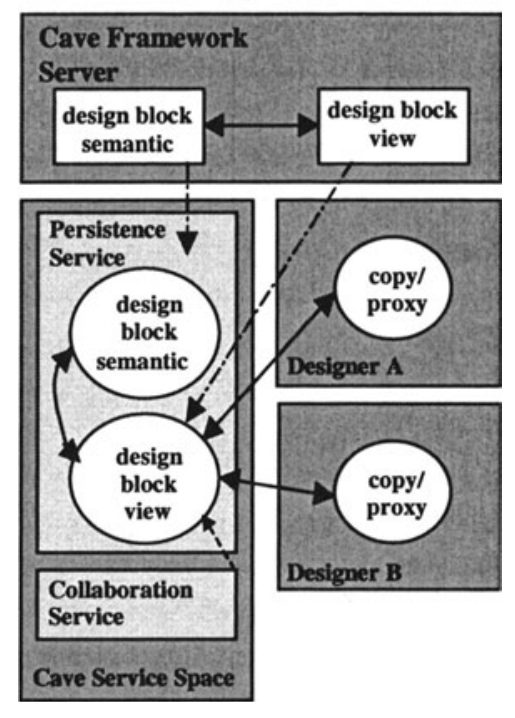

Figure 2. Implemented support for collaboration

colleague. The method is considered synchronous co-located by the timespace taxonomy of collaborative systems, because both developers work at the same time and at the same machine, alternating the control of the keyboard systematically. In the current work, this method was extended and adapted to be used over a network of remote participants, allowing the collaborative work to be done using both text and diagrams. Two major differences were included:

- the participants are not co-located and rely on the network infrastructure to collaborate;

- the collaboration among three or more users is allowed.

For the sake of correctness, the altered methodology was called paar programming, named after the German word paar, which designates a collection of elements. 
In the implemented prototype, the Collaboration Service is responsible for the management of the users and their collaboration groups, each one named paar. In each paar, only one user can have the "keyboard control", or in this case the permission to update the design block the group is working on. The rest of the group will have read-only access to the design block. Several control mechanisms are being implemented to change the control from one user to another, based on consensus among the users, randomic choices and fixed amounts of time - as used by some of the original pair programming enthusiasts.

The transaction-based model is used to make this approach real. When a paar is formed to collaborative build a design block, every user but the leader opens a read-only transaction to that block, which is stored as an object by the Persistence Service. Then, a transaction is opened to the Persistence Service by the paar leader, granting him/her write privileges while write-locking the design object for the other paar members. Every update done by the leader is broadcast to the other members using the update/notify pattern. When a leader substitution happens, the leader transaction is closed, the lock is released and he/she opens then a read-only transaction. The next leader opens a read/write transaction, locking the design block, and this procedure loops until the end of the design block edition.

The current prototype uses Jini/JavaSpaces [13] and Ozone [19] to implement the service space, hosting the persistency and collaboration services.

To validate the proposed approach, several experiences are being done over the prototyped environment. Both kinds of design description languages and diagrams - are being used, and a IRC-like text-based communication tool is used for the coordination of the design team. Further work should be done to provide a voice-over-IP channel for real conversation among the designers, enriching even more the collaboration.

About the collaboration experiences observed up to now, it is important to notice that the initial resistance of the designers to work in group using the transaction-based approach is quickly overcome. As the designers get used to the pace of the control change, they start to enjoy the possibility of having from time to time an "outside view" of the design while the colleague has the control of the design block under development. Most of the advantages of the Pair Programming methodology found in the literature could be also noticed, such as:

- productivity increase, because one designer is much more focused when are there other colleagues depending on his/her work;

- quality improvement, because the design blocks are reviewed by several people, and usually each one has better perception to a particular detail; 
- experience transfer, because the methodology motivates the designer to learn from the expertise of his/her colleagues.

\subsection{Design Tool Prototypes}

In order to make the collaborative system design possible, two design entry tools were prototyped, based on the Cave2 primitives. For diagrammatic entry, a flexible editor called BLADE was created [20]. BLADE allows the collaborative creation and edition of hierarchical block diagrams. The editor supports multiple visual languages, so it can be used in all the cycles of the design, from the functional specification - using UML, for instance - to the structural design - using logic schematics.

The diagrammatic entry is not so effective in the cycles of the design flow where the detailed behavior and structure of the system are input. In those cases, the graphical entry is only performed to define the interface of a particular module with the rest of the system. The actual specification of the module is done using an HDL. To support the collaboration on textual entry, a collaborative text editor named Homero was prototyped [21]. Homero can be executed as a standalone tool, for small projects, or executed as a plug-in to BLADE, when of the programming of the behavior of a particular module of a larger design.

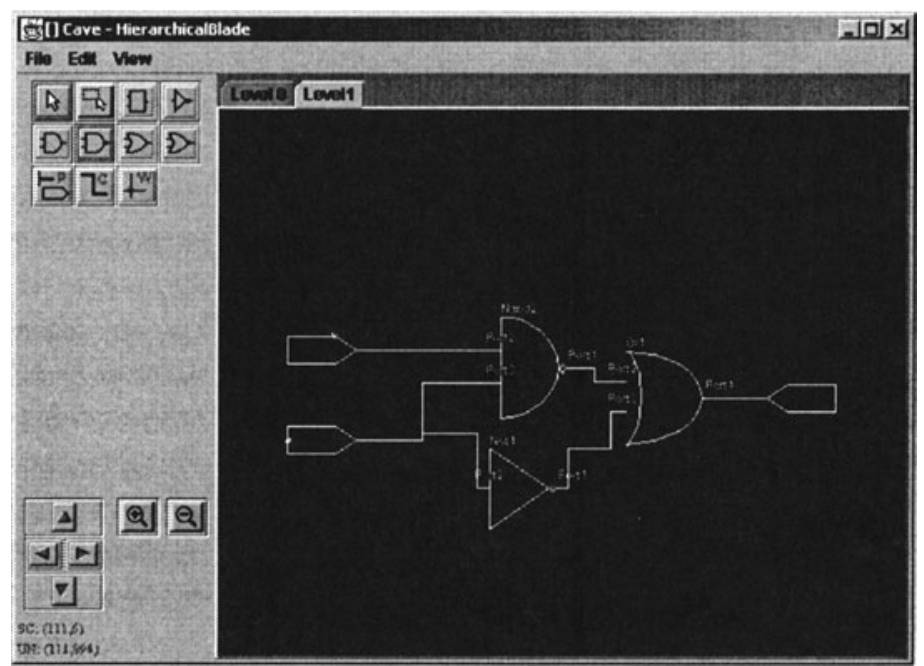

Figure 3. BLADE Graphical User Interface

Both the tools work over instances of the design representation primitives, allowing the designer to share the design data using the Persistence service on the Cave Service Space. Due to its peculiar 
characteristics, the textual data can be shared synchronously in the visually coupled model only. At this time, the effort to provide separate modeling for the text rendering - indentation, font type, size, color, etc. - in order to allow visually decoupled collaboration over text could not be taken.

\section{CONCLUSIONS}

This paper presented a design environment which allows the collaborative design over a computer network. The major concern of this paper is on human aspects of the design process, so it focused on the interaction and collaboration among designers, detailing the technological infrastructure developed to make such synergy possible.

The current prototype of the environment is executed over the Internet, using and extending the infrastructure of the Cave2 Design Framework. The major upgrades to the Cave2 infrastructure were on (1) the separation of semantic and visualization on the design data modeling; (2) keeping consistency after the separation using a pattern for state update/notification; and (3) the inclusion of a transaction-based service for controlling the access to the design data by a set of concurrent designers.

Those extensions to the Cave2 Framework are the contributions of this paper to the state of the art in collaborative design automation environments.

An extension to Pair Programming - a widely used collaboration methodology - was also described in this paper. The extension was necessary because of the nature of the collaboration we were looking for (among larger groups of distributed users), changing some of the basics of the Pair Programming methodology. So, for the sake of correctness the new approach received a new name: Paar Programming, once it changed the allowed number of participants in the collaboration, as well as introduced the possibility of remote collaboration. The implementation and the application of such methodology is another contribution of this paper.

The most important contribution, though, may be the experience on the collaborative work between designers. The need of productivity boost in the hardware industry, together with the shortage of hardware engineers, creates a momentum where the levels of abstraction on the design process should be risen and a new profile for engineering teams - able to do collaborative design through a systemic approach - should be followed.

So, this work intends to be the environment to allow such collaboration, as well as the training environment for the next generation of professionals, which will have the opportunity to learn the design process in a systemic level right from the start, by sharing the experiences of those colleagues who had to learn during the transition from the previous paradigm. 
7. ACKNOWLEDGEMENT

The authors wish to acknowledge and thank Lisane Brisolara, Sandro Sawicki and Emerson Hernandez for their contributions on the design and implementation of the prototypes described in Section 5.2.

\section{REFERENCES}

[1] D. Gajski et al. "The SpecC Methodology". http://www.ics.uci.edu/ specc

[2] S. Swan et. al. "Functional Specification for SystemC 2.0". http://www.systemc.org

[3] D. Davis et. al. "Forge-J: High Performance Hardware from Java". http://www.xilinx.com/forge/forge.htm

[4] DESICS Division. "Ocapi-xl". http://www.imec.br/ocapi

[5] T.J. Barnes et al.. Electronic CAD Frameworks, Kluwer Academic Publishers, 1992.196p.

[6] L.S. Indrusiak and R.A.L. Reis. "A WWW approach for EDA tool integration", Proceedings of the X SBCCI, Gramado, 1997.

[7] L.S. Indrusiak and R.A.L. Reis. "A Case Study for the Cave Project", Proceedings of the XI SBCCI, IEEE Computer Society Press, Armacao de Buzios, 1998.

[8] L.S. Indrusiak and R.A.L. Reis. "3D integrated circuit layout visualization using VRML", Future Generation Computer Systems, Elsevier, v.17, n.5, 2001, p.503 - 511.

[9] J. L. Fragoso, F. Moraes and R. Reis. "WTROPIC: a macro-cell generator on World Wide Web", Proceedings of the XIII SBCCI, IEEE Computer Society Press, Manaus, 2000.

[10] L.S. Indrusiak and R.A.L. Reis. " From a Hyperdocument-Centric to an Object-Oriented Approach for the Cave Project", Proceedings of the XIII SBCCI, IEEE Computer Society Press, Manaus, 2000.

[11] R. Johnson and B. Foote. "Designing Reusable Classes", Journal of Object-Oriented Programming, Vol 1 (2), 1988, pp. 22-35.

[12] E. Gamma et al. Design Patterns - Elements of Reusable Object-Oriented Software, Addison-Wesley, 1995. $395 \mathrm{p}$.

[13] E. Freeman, S. Hupfer and K. Arnold. JavaSpaces: principles, patterns, and pratice, Java Series, Addison Wesley, 1999.

[14] C. Kobryn. "UML 2001: A Standardization Odyssey", Comm. ACM, 42 (10), 1999.

[15] J. Ellsberger, D. Hogrefe and A. Sarma. SDL - Formal Object-Oriented Language for Communication Systems, Prentice Hall, 1997, 312 p.

[16] F. Brglez and H. Lavana. "A Universal Client for Distributed Networked Design and Computing". In Proceedings of the 38th Design Automation Conference, Las Vegas, 2001.

[17] D.R.Kirowski, M. Potkonjak and M. Drinic. "Hypermedia Aided Design". In Proceedings of the 38th Design Automation Conference, Las Vegas, June 2001.

[18] L. Williams, R.R. Kessler. "All I Really Need to Know about Pair Programming I Learned In Kindergarten", Comm. of the ACM Vol. 43 No. 5, 2000. P. 108-114.

[19] G. Mueller and F. Braeutigam. "Tutorial: Getting started with ozone". The Ozone Database Project, 2000. http://www.ozone-db.org

[20] L. B. Brisolara, L. S. Indrusiak, R. Reis. "An Hierarchical Schematic Editor to WWW". In Proceedings of the I Microelectronics Students Forum, Pirenopolis, 2001.

[21] É. B. Hernandez, S. Sawicki, L. S. Indrusiak, R. Reis. "Homero - Um editor VHDL Cooperativo via Web". In Proceedings of the IWS 2001 - VII Workshop Iberchip, Montevideo, 2001. 